The errors in the total difference quantities are around $0.1 \mathrm{kcal} \mathrm{mole}^{-1}$, but errors in the component thermochemical quantities on the right side may be considerably larger. The theoretical rate relations and detailed vibrational frequency assignments for radical and decomposition complexes employed in the calculations are similar in nature to the models previously described and may be found in Refs. 1(e) and 2.

Since the relevant $\Delta H_{f 0}{ }^{\circ}$ are known, ${ }^{4}$ the absolute values, $E_{0}{ }^{t 55 \mathrm{D} M H 2}=29.57 \mathrm{kcal}$, and $E_{\mathrm{min}}=39.85 \mathrm{kcal}$, may be calculated from expressions given in Ref. 1(b). The critical energy for the neo-pentyl addition to the double bond of $c \mathrm{~B} 2$ relative to $t \mathrm{~B} 2$ is $E_{c}($ neoP, $c \mathrm{~B} 2)-$ $E_{c}($ neoP, $t \mathrm{~B} 2)=0.55 \mathrm{kcal} \mathrm{mole}^{-1}$. Also, the difference in critical energies for methyl addition to the number $3 \mathrm{C}$ atom of $55 \mathrm{DMH} 2$ isomers is

$$
\begin{aligned}
E_{c}\left(\mathrm{Me}\left[\mathrm{C}_{3}\right], c 55 \mathrm{DMH} 2\right)-E_{c}\left(\mathrm{Me}\left[\mathrm{C}_{3}\right], t 55 \mathrm{DMH} 2\right) \\
=0.85 \mathrm{kcal} \mathrm{mole}^{-1} .
\end{aligned}
$$

Previously, ${ }^{\text {lb,1c }}$ the critical energy difference

$$
E_{c}(\mathrm{R}, c \mathrm{~B} 2)-E_{c}\left(\mathrm{Me}\left[\mathrm{C}_{3}\right], t \text {-2-alkene }\right)=0.6 \mathrm{kcal} \mathrm{mole}^{-1}
$$

was described for $\mathrm{R}$ is ethyl or larger, and $t$-2-alkene is trans-2-pentene or larger. Thus, on taking the difference $\Delta E_{c}$ in Eq. (3) to be $0.6 \mathrm{kcal} \mathrm{mole}{ }^{-1}$, then $D_{0}{ }^{\circ}(\mathrm{Me}-\mathrm{H})-$ $D_{0}^{\circ}($ neoP-H $)=3.9 \mathrm{kcal}^{\mathrm{mole}}{ }^{-1}$. Previous competitive reaction studies led to $D_{0}{ }^{\circ}(\mathrm{Me}-\mathrm{H})-D_{0}{ }^{\circ}(\mathrm{Et}-\mathrm{H})=5.70$ kcal mole ${ }^{-1}$ so that $D_{0}^{\circ}($ neoP-H $)$ exceeds $D_{0}^{\circ}(\mathrm{Et}-\mathrm{H})$ by $1.8 \mathrm{kcal} \mathrm{mole}^{-1}$ from our studies ( $\mathrm{cf} .1 .4 \mathrm{kcal} \mathrm{mole}^{-1}$ from $\left.\mathrm{Kerr}^{5}\right)$. Bond dissociation energies, $D_{0}(\mathrm{R}-\mathrm{H})$, for $\mathrm{R}$ is methyl, ethyl, $n$-propyl, iso-propyl, and neo-pentyl, have now been fixed by competitive reaction studies at 102.7, 97.0, 96.2, 92.8, and $98.8 \mathrm{kcal} \mathrm{mole}^{-1}$, respectively. At $298^{\circ} \mathrm{K}$, the corresponding values are 104.3 , 98.6, 97.7, 94.3, and $100.3 \mathrm{kcal} \mathrm{mole}^{-1}$, respectively. The estimated accuracy of these $D^{\circ}$ quantities is within $\pm 1 \mathrm{kcal}$ on an absolute basis; the relative errors should be less.

* This work was supported by the Office of Naval Research.

$\dagger$ NSF Predoctoral Trainee.

+ NSF Predoctoral Fellow.

1 (a) D. C. 'Tardy, B. S. Rabinovitch, and C. W. Larson, J. Chem Phys. 45, 1163 (1965); (b) C. W. Larson, B. S. Rabinovitch, and D. C. Tardy, ibid., 47, 4570 (1967); (c) C. W. Larson, D. C. Tardy, and B. S. Rabinovitch, ibid, 49, 299 (1968); (d) C. W. Larson and B. S. Rabinovitch, ibid., 50, 871 (1969); in Eq. (7) the ratio in parenthesis should be inverted and the numbers 2 and 5 in the line below Eq. (7) should be interchanged. (e) See. C. W. Larson, Ph.D. thesis, University of Washington, Seattle, Wash., 1969 for further details.

${ }^{2}$ C. W. Larson, B. S. Rabinovitch, and E. A. Hardwidge (unpublished).

${ }^{3}$ R. A. Marcus and O. K. Rice, J. Phys. Colloid Chem. 55, 894 (1951).

${ }^{4}$ Heats of formation at $298^{\circ} \mathrm{K}$ were calculated using the technique described in J. D. Rockenfeller and F. D. Rossini, J. Phys. Chem. 65, $267(1961)$ and corrected to $0^{\circ} \mathbf{K}$ using $H_{208}{ }^{\circ}-H_{0}^{\circ}$ estimated from J. L. Franklin, Ind. Eng. Chem. 41, 1070 (1949). Results are kilocalories mole $\left.{ }^{-1}\right): \Delta H_{f 0}^{\circ}(c 55 \mathrm{DMH} 2)=$ $-14.65 ; \Delta H_{f 0}^{\circ}(t 55 \mathrm{DMH} 2)=-15.85 ; \Delta H_{f 0}^{\circ}(2,4,4$-trimethylhexane $)^{\prime}=-43.71 ; \Delta H_{f 0}{ }^{\circ}(3,5,5$-trimethyl-1-hexene $)=-15.70$. Also, $\Delta H_{f 0}{ }^{\circ}$ (neopentene) $=-31.30$ from F. D. Rossini, Selected Values of Physical and Thermodynamic Properties of Hydrocarbons
(Carnegie Press, Pittsburgh, Pa., 1953) was used. Using previously adopted or determined values ${ }^{1 d}$ for the remaining required thermodynamic properties, then $E_{0}{ }^{155 \mathrm{DMH} 2}[$ see Eq. (8), Ref. $1(\mathrm{~b})]$ the critical energy for formation of $t 55 \mathrm{DMH} 2$ and $E_{\mathrm{min}}$ [see Eq. $(7)$, Ref. $1(\mathrm{~b})]$ the minimum energy of the formed vibrationally excited radical $\left(355 \mathrm{TMH} 2^{*}\right.$ ) were obtained.

${ }^{5}$ J. A. Kerr, Chem. Rev. 66, 465 (1966).

\section{Heat Capacity of Silver Iodide. III. An Order-Disorder Transition}

\author{
C. M. Perrott and N. H. Fletcher \\ Department of Physics, University of New England, \\ Armidale, N.S.W., A ustralia \\ (Received 30 September 1968)
}

In the first two papers of this series, ${ }^{1,2}$ to be referred to as I and II, we reported measurements of the heat capacity of silver iodide over the range $50^{\circ}-360^{\circ} \mathrm{C}$ and proposed a theoretical model to account for the anomalously high heat capacity of stoichiometric $\alpha$-AgI. This model was based on the assumption of an orderdisorder transition between $N$ silver ions and $2 N$ silver ion vacancies distributed over the $3 N$ equivalent cluster sites of six interpenetrating simple cubic lattices which may be used to describe the $\alpha$-AgI structure. No such transition was observed within the range of the measurements and it was assumed to occur somewhere between $360^{\circ} \mathrm{C}$ and the melting point, $550^{\circ} \mathrm{C}$.

These measurements have now been extended to $480^{\circ} \mathrm{C}$ and the order-disorder transition has been identified at $430^{\circ} \mathrm{C}$. In addition, further information now makes it possible to refine our model for the cluster sites and to make a more significant comparison between theory and experiment.

Our experimental results are summarized in Fig. 1 and show the typical form of an order-disorder transition. At the critical temperature $430^{\circ} \mathrm{C}$, however, not only does the heat capacity change discontinuously, but there is a latent heat amounting to $300 \pm 50$ cal/mole. This implies a discontinuous change in the order parameter at the critical temperature, which was, in fact, a feature of the theory presented in II.

In seeking numerical values for the theory in II, we made the assumption that the lowest energy is associated with the central $(b)$ sites in each cluster, the surrounding $(d)$ and $(h)$ sites having higher energies. It has been pointed out to us by P. D. Greene that this is most unlikely, and recent powder-diffraction data by Burley ${ }^{3}$ indicates that the group occupancies of $(b)$, $(d)$, and $(h)$ sites at $430^{\circ} \mathrm{C}$ are as $2.4: 26.4: 72$. The $(h)$ sites thus have lowest energy, with $(d)$ and $(b)$ sites lying above them by approximately 0.26 and 1.73 $\mathrm{kcal} /$ mole, respectively. We have evaluated the contribution to the specific heat of the vibrational motion of silver ions in these cluster wells, using a different approximation for the potential from that in II in order to avoid supernumary wells, and find that it is 
FIG. 1. Molar heat capacity of annealed stoichiometric silver iodide: (a) Typical experimental results, those below $360^{\circ} \mathrm{C}$ being derived from the previous work ${ }^{1}$ and the overlapping results above $300^{\circ} \mathrm{C}$ representing the present work; (b) results of Lieser ${ }^{4}$ (c) theory for $\alpha$-AgI.

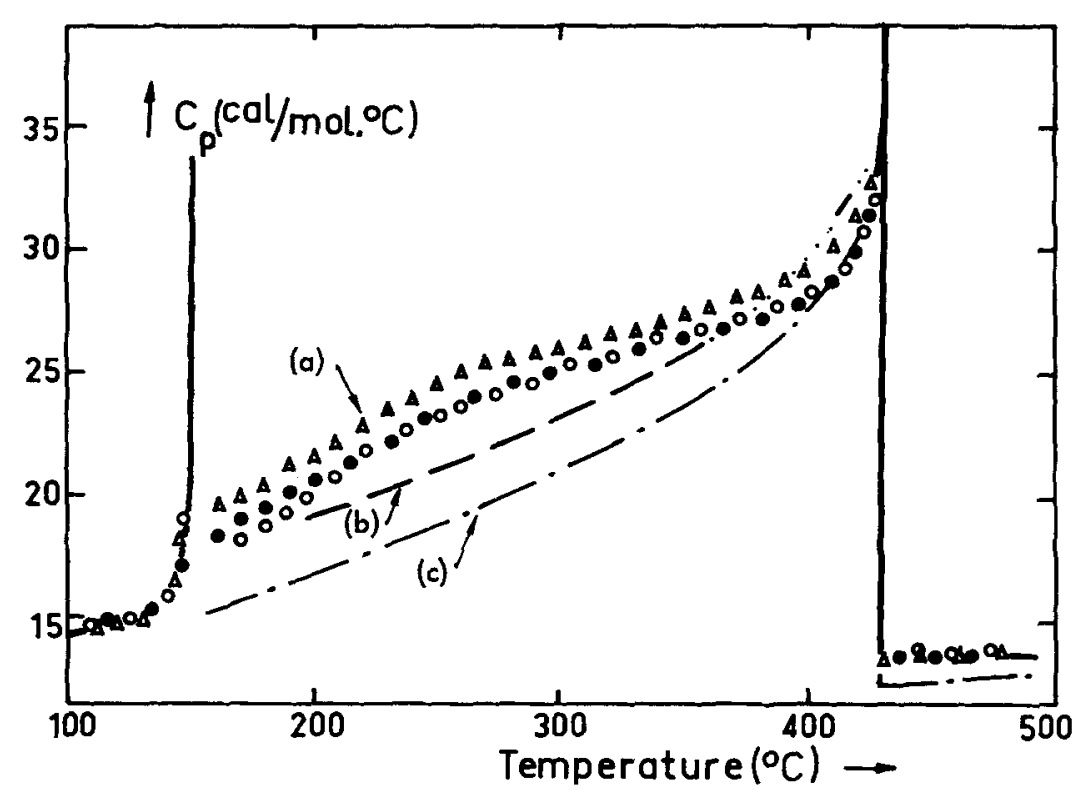

less than the normal value $3 R$ by about $0.70 R$ at $200^{\circ} \mathrm{C}, 0.70 R$ at $350^{\circ} \mathrm{C}$, and $0.02 R$ at $500^{\circ} \mathrm{C}$.

Before presenting the theoretical heat-capacity curve, it is useful to point out that the whole orderdisorder problem in $\alpha$-AgI can be simplified if the $3 \mathrm{~N}$ equivalent cluster sites are regarded as comprising not six simple cubic lattices but rather three body-centered cubic lattices. Each such lattice then has the same symmetry as does the iodide ion lattice and it is natural to expect silver ions to occupy just one of these lattices in the ordered state, leaving the other two vacant. It is then no longer necessary to make any special assumptions about the variation of interaction energy with distance because the nearest-neighbor interactions considered always occur over a separation $\frac{1}{2} a_{0}$ and the number of nearest neighbors $z^{i j}$ is constant. On this sublattice model the results are identical with those presented in II but the physical foundations seem more secure.

Finally, to calculate the heat capacity of $\alpha$-AgI we obtain the configurational heat capacity in terms of absolute temperature from the correspondence of critical temperatures $\tau=0.915$ from II and $T=703^{\circ} \mathrm{K}$ as observed. This sets the ordering energy $v=k T / \tau$ as $1.54 \mathrm{kcal} / \mathrm{mole}$. To this we add the vibrational contribution from silver ions referred to above and the normal $3 R$ from iodide ions. The disordering contribution from iodide ions discussed in II is omitted since its existence is uncertain and it is certainly overestimated by the evaluation suggested in II. The final result is shown as curve (c) in Fig. 1 , where it is compared with our own measurements (a) and those of Lieser ${ }^{4}$ (b), after conversion from $C_{v}$ to $C_{p}$ as discussed in $\mathrm{I}$.

The agreement between theory and experiment for heat capacity is not at all exact but is reasonably satisfactory in view of the zeroth-order nature of the order-disorder theory used in the derivation. The calculated value of the latent heat at the transition is $110 \mathrm{cal} / \mathrm{mole}$, which is very much less than the experimental value $300 \pm 50 \mathrm{cal} / \mathrm{mole}$, but again moderately satisfactory in view of the approximations involved.

The authors wish to thank the Australian Research Grants Committee for financial support and the Commonwealth Scientific and Industrial Research Organisation for a scholarship award for one of us (C.M.P.).

${ }^{1}$ C. M. Perrott and N. H. Fletcher, J. Chem. Phys. 48, 2143 (1968)

${ }^{2}$ C. M. Perrott and N. H. Fletcher, J. Chem. Phys. 48, 2681 (1968)

${ }^{3}$ G. Burley, Acta Cryst. 23, 1 (1967).

${ }^{4}$ K. H. Lieser, Z. Physik. Chem. (Frankfurt) 2, 238 (1954).

\section{Approximate Hartree-Fock Energies of the $1^{1} S$ and $2^{3} S$ States of the Helium Atom Using Three-Parameter Orbitals Generalized from Slater and Gaussian Functions}

\author{
Robert H. Carrier and F. L. PIlar \\ University of New Hampshire, Department of Chemistry, \\ Parsons Hall, Durham, New Hampshire
}

(Received 27 September 1968)

During the course of recent investigations on diatomic molecules we have had occasion to carry out certain calibration calculations on the helium atom using trial wavefunctions constructed from antisymmetrized products of spin orbitals whose spatial portions are of the general form

$$
\phi_{i}\left(\alpha_{i}, n_{i}, m\right)=N r^{n_{i}} \exp \left(-\alpha_{i} r^{m}\right),
$$

